\title{
Associated production of $J / \psi$ and $\Upsilon$ mesons and the prospects to observe a new hypothetical tetraquark state
}

\author{
Sergey Baranov ${ }^{1, \star}$ \\ ${ }^{1}$ P.N. Lebedev Institute of Physics, 53 Lenin Avenue, Moscow 119991, Russia
}

\begin{abstract}
We propose a new mechanism for prompt simultaneous production of $J / \psi$ and $\Upsilon$ mesons in high energy hadronic collisions. The process is considered as a perturbative production of $B_{c}^{(*)}$ mesons $g+g \rightarrow B_{c}^{(*)}+\bar{B}_{c}^{(*)}$ followed by a long-distance final state interaction that rearranges the quarks to form $J / \psi$ and $\Upsilon$ mesons. Passing from $B_{c}^{(*)}+\bar{B}_{c}^{(*)}$ configuration to $J / \psi+\Upsilon$ configuration may proceed via a hypothetical resonance state, the tetraquark. The goal of this work is to examine whether the respective cross section is large enough to encourage a direct search for the tetraquark at the LHC conditions (yes), and whether this hypothesis can help to explain recent D0 data without assigning an unusually low value to $\sigma_{\text {eff }}$ in the double parton scattering mechanism (no).
\end{abstract}

\section{Introduction}

After a number of discoveries revealing the existence of tetra-quark mesonic states and penta-quark baryonic states, one should not be surprised with finding yet more particles in these families. For example, it would not be unreasonable to suggest the existence of a $b \bar{b} c \bar{c}$ state composed of four heavy quarks. Its inner structure can be described as a hadron molecule $B_{c}+\bar{B}_{c}$, or a hadro-quarkonium $\Upsilon+J / \psi$, or, most probably, the quantum superposition of the two. Such a state must then be naturally connected to the $B_{c}+\bar{B}_{c}$ and $\Upsilon+J / \psi$ states, so that these quark configurations could easily transform into one another if the invariant energy of the system is close to the $b \bar{b} c \bar{c}$ resonant mass. The hypothetical Tetraquark can thus be observed experimentally via its four-lepton decay in the $\Upsilon+J / \psi$ channel. The goal of this note is to examine whether the respective cross section is large enough to encourage a direct detection of the tetraquark at the LHC conditions, and whether this hypothesis can help to explain the D0 data without assigning an unusually low value to $\sigma_{\text {eff }}$ in the double parton scattering (DPS) mechanism.

\section{The model}

We understand the production mechanism as a two-step process. The first step is a purely perturbative one and refers to the production of a $B_{c} \bar{B}_{c}$ or $B_{c}^{*} \bar{B}_{c}^{*}$ pair in a short-distance interaction. At the second step, the four heavy quarks get reshuffled in a long-distance final state interaction thus forming the

^e-mail: baranov@sci.lebedev.ru 
$J / \psi$ and $\Upsilon$ mesons. We start with calculating the production of $B_{c}$ pairs in the gluon-gluon fusion subprocess at $O\left(\alpha_{s}^{4}\right)$ :

$$
g+g \rightarrow B_{c}^{(*)}+\bar{B}_{c}^{(*)}
$$

Here we follow the standard QCD Feynman rules and basically repeat our earlier work [1]. The only innovation is in using the $k_{t}$-factorization approach [2]. We see the advantages of the latter in the ease of including higher-order corrections, which can be taken into account in the form of $k_{T}$-dependent parton densities. These corrections significantly modify the event kinematics; in particular, they give non-zero transverse momentum to the $B_{c}$ pair and destroy the back-to-back azimuthal correlations. Technically, the computation of Feynman diagrams implies a modified gluon spin density matrix, which is now taken in the form [2-4] $\overline{\epsilon_{g}^{\mu} \epsilon_{g}^{* v}}=k_{T}^{\mu} k_{T}^{v} /\left|k_{T}\right|^{2}$, where $k_{T}$ is the component of the gluon momentum perpendicular to the beam axis. In the collinear limit, when $k_{T} \rightarrow 0$, this expression converges to the ordinary $\overline{\epsilon_{g}^{\mu} \epsilon_{g}^{* v}}=-\frac{1}{2} g^{\mu \nu}$. In all other respects the calculations are standard.

Throughout this note, the calculations are based on the following parameter setting. The charmed and bottom quark masses are set to one half of the $J / \psi$ and $\Upsilon$ masses, respectively: $m_{c}=m_{\psi} / 2=$ $1.55 \mathrm{GeV}$ and $m_{b}=m_{\Upsilon} / 2=4.8 \mathrm{GeV}$ (we also have to obey $m_{B_{c}}=m_{b}+m_{c}$ ); the factorization and renormalization scales are set equal to each other and $\mu_{F}^{2}=\mu_{R}^{2}=\hat{s} / 4$ where $\hat{s}$ is the invariant energy of the partonic subprocess (this choice is Lorentz-invariant and is symmetric with respect to both final state particles); we use $A 0$ parametrization for the unintegrated ( $k_{T}$-dependent) gluon density taken from Ref. [5]; and we use radial wave functions of $B_{c}^{(*)}$ mesons $\left|\mathcal{R}_{B_{c}}(0)\right|^{2}=1.2 \mathrm{GeV}^{3}$ taken from the potential model of Ref. [6]. Variations in the scales $\mu_{F}^{2}$ and $\mu_{R}^{2}$ or using the $A+$ or $A-$ gluon parametrizations instead of $A 0$ may increase or decrease the estimated production rate by a factor of 2 or 3. Note however, that the aim of this note is not in giving exact predictions for an unknown particle, but rather in giving some reasonable hints that would help to make a decision on whether a dedicated search for a new particle is worth starting.

For an illustrative example we consider the kinematics of LHCb experiment, as it has no complications and no reduction of the cross section coming from the cuts on the muon transverse momentum. Note also that the $k_{t}$-factorization approach was able to perfectly describe the recent LHCb data on double $J / \psi$ production [7]; the matrix element of the latter process can be obtained from that of $B_{c} \bar{B}_{c}$ case by just replacing $m_{b}$ with $m_{c}$.

The $B_{c} \bar{B}_{c}$ invariant mass spectrum calculated for $\sqrt{s}=13 \mathrm{TeV}$ is shown in Fig. 1 . We see that the cross section mostly concentrates at nearby threshold, where the relative quark velocities are not large, thus giving a good chance for final state interactions. As a consequence, the quarks can reshuffle and form $J / \psi$ and $\Upsilon$ mesons:

$$
B_{c}+\bar{B}_{c} \rightarrow b \bar{b} c \bar{c} \rightarrow J / \psi+\Upsilon .
$$

To calculate the transition amplitude of (2) we, strictly speaking, need to know the overlap integrals for the initial and intermediate, and for the intermediate and final state wave functions. For the lack of the information on the tetraquark wave function, we use a semi-qualitative approximation based on the $1 / N_{c}$ topological expansion and quark-hadron duality.

The diagram of Fig. 1 with crossing quark lines is a typical nonplanar diagram in the $1 / N_{c}$ topological expansion [8-10]. Diagrams of this kind violate Okubo-Zweig-Iizuka (OZI) rule and are suppressed by an approximate factor of $1 / N_{c}^{2}$ in comparison with OZI non-violating planar diagrams of the same quark content (the latter refer to the $B_{c}+\bar{B}_{c}$ final states). The suppression factor is easily calculable in our case by taking the projection of the $B_{c}+\bar{B}_{c}$ color state $\delta^{b \bar{c}} \delta^{c \bar{b}}$ onto the $J / \psi+\Upsilon$ color state $\delta^{b \bar{b}} \delta^{c \bar{c}}$; taking the trace of color indices yields a factor of $1 / 8$.

This factor gives the ratio of the production cross sections for two different color configurations, but it tells nothing on whether the two color singlets are bound or unbound. We can appeal here to 

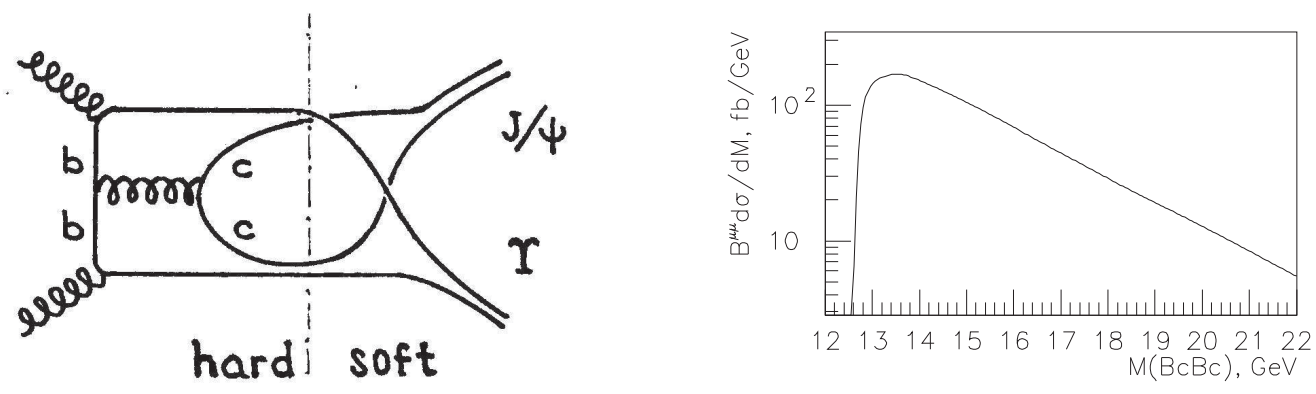

Figure 1. A diagram representing the $J / \psi+\Upsilon$ production mechanism discussed in the text (left panel) and our predictions on the $B_{c} \bar{B}_{c}$ invariant mass ditribution at LHCb at $\sqrt{s}=13 \mathrm{TeV}$ (right panel).

the ideas of quark-hadron duality [11] that the cross section integrated over a continuous spectum must be the same as the cross section summed over a discrete number of resonances. That is, the estimate $\sigma(J / \psi+\Upsilon) \simeq \sigma\left(B_{c}+\bar{B}_{c}\right) / N_{c}^{2}$ shows the correct number of $J / \psi+\Upsilon$ states, and if the hypothetical tetraquark does exisit, this estimate corresponds to the tetraquark production cross section; otherwise, it corresponds to the unbound $J / \psi$ 's and $\Upsilon$ 's.

Now we turn to numerical results. The integral production cross section times muon branching fraction amounts to $\sigma\left(p p \rightarrow B_{c} \bar{B}_{c}\right) B r^{\Upsilon \rightarrow \mu \mu} B r^{\psi \rightarrow \mu \mu} \simeq 550 \mathrm{fb}$. By including the muon branchings we also mean that the muons fit the LHCb kinematics $2<\eta(\mu)<4.5$. Then, applying the topological suppression factor we find for the $J / \psi+\Upsilon$ production rate

$$
\sigma(\Upsilon+J / \psi) B r^{\Upsilon \rightarrow \mu \mu} B r^{\psi \rightarrow \mu \mu} \simeq 60 \mathrm{fb},
$$

where the estimated [12-14] four-lepton decay branching is not in contradiction with the naive guess $B r^{b \bar{b} c \bar{c} \rightarrow \mu \mu \mu \mu} \simeq B r^{\Upsilon \rightarrow \mu \mu} B r^{\psi \rightarrow \mu \mu} \simeq 1.510^{-3}$. So, the expected production rate is small, though not discouragingly small, and is at least not smaller than the integral $\Upsilon+J / \psi$ cross section measured by the collaboration D0.

The tetraquark signal should be seen over a background formed by both single (SPS) and double (DPS) parton scattering. First, we briefly discuss the SPS contributions. Contrary to the production of same-flavor meson pairs, the associated production of $J / \psi$ and $\Upsilon$ mesons in the color-singlet mode is not possible at $O\left(\alpha_{s}^{4}\right)$. The leading SPS mechanism refers to a mixed singlet-octet scheme, with $\Upsilon$ formed in the color-singlet mode $g+g \rightarrow \Upsilon+g^{*}$ and $J / \psi$ coming from the virtual (color-octet) gluon fragmentation $g^{*} \rightarrow c \bar{c}\left[{ }^{3} S_{1}^{8}\right] \rightarrow J / \psi$. The fully octet modes are suppressed because of typically much smaller values of the color-octet matrix elements (as compared to the color-singlet wave functions). These contributions can only become important at high $p_{T}$, that is not the main region of the $\mathrm{LHCb}$ phase space. Simulataneous color-singlet production of $P$-wave states $g+g \rightarrow \chi_{b}+\chi_{c}$ is possible at $O\left(\alpha_{s}^{4}\right)$, but is suppressed by lower values of the $P$-state wave functions and, further, by the subsequent radiative transition branching fractions $\chi_{c} \rightarrow J / \psi+\gamma, \chi_{b} \rightarrow \Upsilon+\gamma$. Simultaneous production of $S$ wave color-singlets is possible at $O\left(\alpha_{s}^{6}\right)$; it is suppressed by two extra powers of $\alpha_{s}$ and, especially, by the color algebra, as is explained in detail in Ref. [15]. Besides, one can consider a non-prompt mechanism, i.e., the production of $\Upsilon+b+\bar{b}$ states at $O\left(\alpha_{s}^{4}\right)$ followed by the decays $b, \bar{b} \rightarrow J / \psi+X$. This contribution is suppressed because of small decay branching fraction $\operatorname{Br}(B \rightarrow J / \psi+X) \simeq 310^{-3}$ [16]. All these contributions taken together can hardly bring more than $\sim 2 \mathrm{fb}$, even with the most optimistic choice for the color-octet matrix elements. The dominant background is certainly brought 
by the double parton scattering. Using the simple factorization formula [17]

$$
\sigma_{\text {DPS }}(\Upsilon+J / \psi)=\sigma_{\text {incl }}(\Upsilon) \sigma_{\text {incl }}(J / \psi) / \sigma_{\text {eff }}
$$

along with the previously measured single inclusive $J / \psi$ [18] and $\Upsilon$ [19] cross sections at $\sqrt{s}=7,8$, and $13 \mathrm{TeV}$

$$
\begin{gathered}
\sigma_{\text {incl, } 13 \mathrm{TeV}}(J / \psi) B r^{\psi \rightarrow \mu \mu} \simeq 380 \mathrm{nb}, \\
\sigma_{\text {incl, } 7 \mathrm{TeV}}(\Upsilon) B r^{\Upsilon \rightarrow \mu \mu} \simeq 2.5 \mathrm{nb}, \quad \sigma_{\text {incl, } 8 \mathrm{TeV}}(\Upsilon) B r^{\Upsilon \rightarrow \mu \mu} \simeq 3.3 \mathrm{nb},
\end{gathered}
$$

extrapolating the $\Upsilon$ results to $\sqrt{s}=13 \mathrm{TeV}$ as

$$
\sigma_{\text {incl, } 13 \mathrm{TeV}}(\Upsilon) B r^{\Upsilon \rightarrow \mu \mu} \simeq 6 \mathrm{nb},
$$

and taking $\sigma_{\text {eff }}=15 \mathrm{mb}$ we arrive at

$$
\sigma(\Upsilon+J / \psi) B r^{\Upsilon \rightarrow \mu \mu} B r^{\psi \rightarrow \mu \mu} \simeq 150 \mathrm{fb} .
$$

With smaller values of $\sigma_{\text {eff }}$ suggested by some other measurements $\left(\sigma_{\text {eff }}=5 \mathrm{mb}\right.$ [20] or even $\sigma_{\text {eff }}=$ $2.2 \mathrm{mb}$ [21]) we arrive at accordingly larger estimations of the background. The signal is smaller than the integral background, but can be distinguished from the latter due to different kinematic properties. The resonant peak in the four-lepton invariant mass distribution contrasts with the broad and flat mass spectrum generated by double parton scattering.

Finally, we turn to the data [21] provided by the Collaboration D0 at the Tevatron. The Collaboration reported on the simultaneous production of $J / \psi$ and $\Upsilon$ mesons in $p p$ collisions at $\sqrt{s}=1.96 \mathrm{TeV}$. Both mesons were seen in the muon decay channel, where all muons were required to have transverse momentum $p_{T}^{\mu}>2 \mathrm{GeV}$ and pseudorapidity $\left|\eta^{\mu}\right|<2.0$. The production cross section measured with these requirements was found to be

$$
\sigma(\Upsilon+J / \psi) B r^{\Upsilon \rightarrow \mu \mu} B r^{\psi \rightarrow \mu \mu}=27 \pm 9(\text { stat }) \pm 7 \text { (syst) } \mathrm{fb} .
$$

This number has to be compared with the hypothetical effect of a $c \bar{c} b \bar{b}$ tetraquark. Following the method described above we find for the integral production rate at the D0 conditions $\sigma(p p \rightarrow$ $\left.B_{c} \bar{B}_{c}\right) B r^{\Upsilon \rightarrow \mu \mu} B r^{\psi \rightarrow \mu \mu} \simeq 22 \mathrm{fb}$. This is comparable with the experimental result Eq.(9), but one has to keep in mind that only a fraction of all $B_{c}^{(*)}$ pairs can convert into $\Upsilon+J / \psi$ states. After applying the topological suppression factor we are left with only 1 or $2 \mathrm{fb}$. The flatness of the $\Delta \phi$ distribution seen in the data is yet not incompatible with the tetraquark hypothesis (if the tetraquark mass is around $14 \mathrm{GeV}$; a result of the calculation not shown in the present note), but the absolute production rate probably is. So we conclude that the considered mechanism cannot take credit for the $\Upsilon+J / \psi$ events observed by D0.

\section{Conclusions}

We have considered a new mechanism for prompt simultaneous production of $J / \psi$ and $\Upsilon$ mesons. This mechanism consists in a perturbative production of $B_{c}^{(*)}$ mesons $g+g \rightarrow B_{c}^{(*)}+\bar{B}_{c}^{(*)}$ followed by a long-distance interaction that rearranges the quarks to form $J / \psi$ and $\Upsilon$ mesons. Passing from $B_{c}^{(*)}+\bar{B}_{c}^{(*)}$ configuration to $J / \psi+\Upsilon$ configuration may proceed via a hypothetical resonance state with $b \bar{b} c \bar{c}$ quark content.

We find that the considered mechanism may be strong enough to encourage a direct search for the $b \bar{b} c \bar{c}$ tetraquark at the LHC conditions. At the same time, the estimated production rate at the D0 conditions is insufficient to provide a reasonable explanation for the observed $\Upsilon+J / \psi$ events. Of course, the conclusive evidence could only be provided by an explicit peak in the four-muon mass spectrum. 


\section{Acknowledgments}

This work was supported by the DESY directorate in the framework of Moscow-DESY project on Monte-Carlo implementations in Physics.

\section{References}

[1] S.P. Baranov, Phys. Rev. D 55, 2756 (1997).

[2] L.V. Gribov, E.M. Levin, M.G. Ryskin, Phys. Rep. 100, 1 (1983);

E.M. Levin, M.G. Ryskin, Yu.M. Shabelsky, A.G. Shuvaev, Sov. J. Nucl. Phys. 53, 657 (1991).

[3] S. Catani, M. Ciafaloni, F. Hautmann, Phys. Lett. B 242, 97 (1990); Nucl. Phys. B366, 135 (1991).

[4] J.C. Collins, R.K. Ellis, Nucl. Phys. B360, 3 (1991).

[5] H. Jung, arXiv:hep-ph/0411287;

H. Jung et al., Eur. Phys. J. C 70, 1237 (2010);

H. Jung, http://www.desy.de/ jung/cascade/updf.html

[6] E. Bagan, H.G. Dosch, P. Gosdzinsky, S. Narison, J.-M. Richard, Z. Phys. C 64, 57 (1994).

[7] R. Aaij et al. (LHCb Collab.), arXiv:1612.07451.

[8] Chan Hong-Mo et al., Nucl. Phys. B 86, 470 (1975); Nucl. Phys. B 92, 13 (1975).

[9] C.F. Chew, C. Rosenzweig, Nucl. Phys. B 104, 290 (1976); Phys. Rev. C 41, 263 (1978).

[10] G. Veneziano, Phys. Lett. B 52, 220 (1974); Nucl. Phys. B 117, 519 (1976).

[11] E.C. Poggio, H.R. Quinn, S. Weinberg, Phys. Rev. D 13, 1958 (1976).

[12] M. Karliner, S. Nussinov, J.L. Rosner, arXiv:1611.00348.

[13] Y. Bai, S. Liu, J. Osborne, arXiv:1612.00012.

[14] Z.-G. Wang, arXiv:1701.04285.

[15] S.P. Baranov, W. Schäfer, A.M. Snigirev, A. Szczurek, N.P. Zotov, Phys. Rev. D 87, 034035 (2013).

[16] C. Patrignani et al. (Particle Data Group), Chin. Phys. C40, 100001 (2016).

[17] P. Bartalini et al., arXiv:1111.0469,

H. Abramowicz et al., arXiv:1306.5413,

S. Bansal et al., arXiv:1410.6664.

[18] R. Aaij et al. (LHCb Collab.), JHEP 1510, 172 (2015).

[19] R. Aaij et al. (LHCb Collab.), JHEP 1511, 103 (2015).

[20] V.M. Abazov et al. (D0 Collab.), Phys. Rev. D 90, 111101(R) (2014).

[21] V.M. Abazov et al. (D0 Collab.), Phys. Rev. Lett. 116, 082002 (2016). 